\title{
How Does the Parents-related Factors Exacerbate and Improve Schizophrenia?
}

\author{
Tianying Zhou ${ }^{1, \dagger}$, Yixuan Zhang ${ }^{2, \dagger}$, Zilin Zhu ${ }^{3, *, \dagger}$ \\ ${ }^{1}$ Baylor University, Waco, United States, 76798 \\ ${ }^{2}$ Flint Hill School, Oakton, United States, 22124 \\ ${ }^{3}$ Rutgers, The State University of New Jersey, New Brunswick, United States, 08854 \\ *Zilin Zhu.Email: zz408@scarletmail.rutgers.edu \\ ${ }^{\dagger}$ These authors contributed equally.
}

\begin{abstract}
Understanding the risk factors of Schizophrenia has essential implications for both the effective prevention and management of mental illness conditions. Through analyzing the etiology of schizophrenia, we have found something in common that the existence of both parents plays a looming role in the various risk factors. Although environmental factors are indeed one of the factors contributing to schizophrenia, genes are nonetheless a non-negligible aspect in Schizophrenia according to the high morbidity rate between relatives. In the meantime, personality traits indicate the susceptibility of schizophrenia as well. Certain traits can be commonly found in Schizophrenia patients. The family is the main place for the formation and development of individual personality. Disharmony of the family environment and inappropriate educational methods will affect the formation of individual personality, which will lead to the occurrence of schizophrenia and other related mental illnesses. The socioeconomic status of parents also has impact on the development of childhood schizophrenia, which is demonstrated in a compelling model called Family Stress Model (FSM). This paper aims to help people to obtain a deeper understanding of schizophrenia and realize the importance of parents-related factors throughout the whole process.
\end{abstract}

Keywords: Schizophrenia, Genetics, Inherited Personality, Parenting Style, Childhood Trauma, Socioeconomic Status of Parents

\section{INTRODUCTION}

Schizophrenia is a serious mental illness characterized by incoherent or illogical thought, bizarre behavior and speech, and delusions or hallucinations, such as hearing voice [1]. The World Health Organization (WHO) indicates that there are 20 million people worldwide who are suffering from schizophrenia [2]. Schizophrenia is a chronic mental illness that requires lifelong treatments and care to manage the symptoms since the medical community still lacks the understanding of the pathophysiology of this disorder and the tools for the curative treatments, which means schizophrenia cannot be easily eliminated [3].

\subsection{Etiology of Schizophrenia}

Similar to other kinds of mental disorders, people could be influenced by multiple sources of risk factors during their lifetime that may lead to the occurrence or the development of schizophrenia. In other words, the fixed or exact causes of schizophrenia for patients have not been discovered. Even though the strong genetic component plays an influential role in the occurrence of schizophrenia, researchers also suggested that some potential risk factors can contribute to developing schizophrenia from multiple dimensions including environmental, social psychological factors and so forth [4]. These may include infection in pregnancy, living environment, childhood trauma and substance abuse, etc. [5][6]. The genetic and molecular mechanisms underpinning such an association are only just beginning to be discovered, and the cumulative and interactive natures between these environmental and genetic factors are able to exacerbate schizophrenia in humans [7]. 


\subsection{Clinical Symptoms and Impacts of Schizophrenia}

The symptoms of schizophrenia can be divided into three categories including positive symptoms, negative symptoms and disorders of relating [8]. Positive symptoms include severe hallucinations that dominate the clinical pictures, severe delusions, incoherent or illogical speech and thoughts, repeated bizarre behaviors are present in someone who has schizophrenia. The examples of negative symptoms would include alogia, affective flattening, anhedonia-asociality (inability to experience pleasure or feel social contacts), reduced motivation of work or study are the absence of certain characteristics that normal people usually possess [9]. In addition to these, Richard S.E. Keefe indicated that the impairment in attention, working memory, problem solving, and social cognition etc. can also be discovered in schizophrenia patients [10]. However, one thing that is necessary to clarify is that cognitive-related indicators are just the phenomena occurring among patients with schizophrenia, whereas positive or negative symptoms are used to diagnose individuals with schizophrenia.

Corresponding to the clinical symptoms above, the impacts of schizophrenia could burden patients themselves or even their family members on the worse quality of life and dysfunction on a daily basis. Delusions always make patients have false beliefs of guilt, persecution, or grandeur. Some patients may hide to guard themselves against an imagined persecutor. Hallucinations involve feeling, seeing, tasting, or smelling things that aren't there but hearing voices is the commonest hallucination. From the perspective of effective communication with others, a coherent and logical thought during the conversation is unable for patients to achieve. They normally switch the topics in all of a sudden and remain in a confused or disordered state of mind. The negative symptoms appear across individuals with schizophrenia are more likely to lead to a hard time to function normally and be less committed in social relationships or even withdraw from interpersonal experiences [11]. Isolation from social interaction and being unmotivated to the surroundings may be their most typical choices. More tragic results of schizophrenia include abuses in drugs and alcohol, self-injury or even suicide, etc.

\subsection{Treatments}

Current treatments are mainly dedicated to manage the development of schizophrenia as well as improve daily functions since the causes are too complicated to be fully discovered. The combination of antipsychotic medication and psychotherapy is always the commonest consideration for effectively treating schizophrenia patients [12]. In addition to this, a majority of patients also choose to integrate some other supported psychosocial treatments into the comprehensive care for them for the sake of rehabilitating social skills or daily functioning [13]. Psychosocial therapy for people diagnosed with schizophrenia includes Cognitive Behavioral therapy (CBT), social or vocational skill training, cognition remediation, supported employment or even family psychoeducation that make patients feel less distressed about their psychotic experiences, help to enhance attention, memory or organizational skills, and keep or obtain competitive ability in employments for their subsistence [14]. Psychoeducation for schizophrenia is also the most indispensable treatment not only for patients themselves but also their family members. This kind of family interventions have proved to be one of the most consistently effective treatment modalities available, with $50 \%-60 \%$ reduction in relapse rate over the treatment as usual [15]. By conducting psychoeducation among families with schizophrenic patients, it can offer family members information about schizophrenia and other mental illnesses, illness management, navigating the mental health system, and community resources they might find helpful. Moreover, it can assist family members in learning communication and problem-solving skills in order to let schizophrenic patients accept the disorder and receive the treatments in time. In this case, a positive and beneficial relationship between caregivers and their relatives who are suffering from schizophrenia is nurtured. Although all the treatments mentioned above may not completely cure people with disorganized schizophrenia, they will provide a turning point that leads to a better life as long as the patients persist in having medications and other effective treatments.

Whether before or after the child is the diagnosed with schizophrenia, the presence of family serves as a double-edged sword. Living in the environment provided by their family members may be one of the pivotal factors in stimulating the development of this mental illness. However, the family support will also function as preventing the relapse of the development of schizophrenia to some extent. This paper is prone to reinterpret the information and impacts of these risk factors from the perspective of the family. Through exploring the role that the family functioned in improving patients' conditions, it is possible to have a further effective discussion about plausible and feasible treatment or intervention that both parents could carry out based on the individualized pathology of schizophrenia.

\section{GENETICS}

As genes being the most decisive factor in human bodies, doctors have suspected that some genes have contributed to the diagnosis of schizophrenia; in other words, aside from being a stress-related mental disease, genetic defects could also lead to psychotic diseases like 
schizophrenia. The genes that cause Schizophrenia could either be inherited from parents or comes from gene mutations.

\subsection{Inheritance}

Biological families partially reflect the possibility of having schizophrenia. While having a close relative who is diagnosed with schizophrenia does not necessarily mean that this person has to have schizophrenia, an individual having a family member with schizophrenia displays a higher risk of developing this disorder [16]. According to the Abridged Weinberg Method, a closer relationship with schizophrenia patients signifies a higher rate of getting schizophrenia. The morbidity rate increases as one's relationship with schizophrenia patients get closer. A direct relative of the schizophrenia patients often exhibits a higher morbidity rate. As studies have shown, the most serious patients of schizophrenia's inheritance are monozygotic co-twins, also known as identical twins who share the exact same genes, whose morbidity rate is as high as 85.8 percent. However, blood relationship is not the only indication of schizophrenia: staying close with a schizophrenia patient - for instance, being a step-relative or spouse also increases the possibility of being diagnosed with schizophrenia with a percentage of 1.8 percent.[17] While close relationships do have some effects on schizophrenia, their influences are significantly smaller than those of relatives. Thus, inheritance's impacts ought not be ignored.

\subsection{Particular Gene}

With the significant increase of schizophrenia's morbidity rate within relatives, the role that genes play in schizophrenia is not negligible. Researchers have suspected a number of genes that might contribute to the disease's liability, while only COMT (catechol-O-methyltransferase) and NRG1(neuregulin 1) stand out from those other suspected genes since researchers failed to gain positive conclusions from the other genes. NRG1 became a susceptibility gene for schizophrenia in 2002 [18]; Munafò, et al. published a paper in "Nature" in 2006, identifying the correlation between NRG1 and schizophrenia, yet admit that further research is needed [19]. However, a later publication in 2015 re-evaluated these genes candidates and came up with a conclusion that these genes and studies related to them do not provide adequate evidence to connect them to the etiology of schizophrenia.[20] Even though the scientists have not yet possessed sufficient understanding of the genetic causes of Schizophrenia, the early suppositions and research set the stage for the future studies.

\section{PERSONALITY TRAITS}

Parents' personalities are useful indicators of their progeny's mental states and developments. Some typical personalities are prone to mental disorders like Schizophrenia; thus, through inheritance, their offspring might possess the same personality traits as well. As the result, personalities inherited from parents also have connections with the diagnosis of schizophrenia considering that some personality traits are more vulnerable to psychopathic diseases.

\subsection{Specific Temperaments}

Over decades of continuous research on the correlation between personality and Schizophrenia, researchers have figured out that several distinctive personalities indicate a higher risk of Schizophrenia. According to the Temperament and Character Inventory (TCI), which is an instrument for personality assessment, Schizophrenia patients exhibit a higher score on harm avoidance (HA) and self-transcendence (ST) than the control group, while have a lower score on novelty seeking (NS), reward dependence (RD), self-directedness (SD), and cooperativeness (CO). Among these traits, an increase in $\mathrm{HA}$ is further supported by a study that analyzes the correlation between schizophrenia patients (and their relatives) and the general population. In a study published in 2012, researchers compare schizophrenia patients and presumed carriers' calculated HA score with the control group.[21] The result shows the first two groups both have remarkably higher scores on $\mathrm{HA}$; thus, HA is suspected to be an underlying character vulnerability to Schizophrenia. These studies suggest that schizophrenia patients usually display unique temperaments and personality traits compared to the general population. In other words, certain inherited genes are likely to increase the possibility of having schizophrenia.

\section{PARENTING STYLE}

Parenting style has a non-negligible influence on children's personality development, mental health and social adaptability, and is closely related to the occurrence of certain mental illnesses. Appropriate parenting style and a good family environment are necessary conditions for the healthy growth of children. In recent years, many studies have shown that an inappropriate parenting style of parents may be closely related to their children's mental illness. A number of studies have found that compared with the control group, patients with schizophrenia have significant differences in family parenting styles. The family parenting style of patients with schizophrenia tends to be rejection, dictatorship, humiliation and excessive control [22][23]. Researchers studied the relationship 
between parenting styles and the prevalence of schizophrenia. Different scholars have put forward different opinions. Some believe that poor parenting methods lead to lower self-efficacy in children, which has a negative effect on rehabilitation [24]. Some scholars also believe that evidence shows that negative parenting styles such as rejection, brutality, and humiliation are significantly related to high neuroticism, and positive parenting styles such as acceptance, warmth, and respect are significantly related to high agreeability and high extroversion. Moreover, excessively controlled parenting methods can cause male subjects to show high injury avoidance, low persistence and low cooperation. The over-controlled parenting style of female subjects showed high harm avoidance and low self-direction [25][26] also found that parents' warm upbringing style is significantly related to their children's high self-discipline. While, parental refusal and excessively controlled parenting styles are significantly related to high mentality and low self-discipline. Therefore, parenting styles may shape the personality of children, thereby increasing or decreasing their probability of suffering from schizophrenia [27]. In addition, Thimm [22] found that the poor interaction between parents and children is the cause of early maladaptive schemas, which is related to the incidence of personality disorders in children.

\section{CHILDHOOD TRAUMA}

Many studies support that childhood trauma is related to schizophrenia. Early research and meta-analysis confirmed that childhood trauma is related to the onset of schizophrenia. Studies have shown that childhood trauma can increase the risk of psychosis by three times [28]. Childhood Trauma in Schizophrenia: Current Findings and Research Perspectives. Frontiers in neuroscience [29], found that patients with schizophrenia had higher scores for emotional neglect and physical neglect, and lower utilization scores for physical abuse and subjective support. Bruni [30] refined the types of childhood trauma, and proposed that among the types of childhood trauma suffered by schizophrenia patients, cannabis abuse, loneliness, running away from home, mental abuse, physical abuse and schizophrenia are more related.

Studies have shown that patients with childhood trauma not only have more prominent symptoms, but also have a poorer prognosis and are more difficult to treat. This includes functional recovery and medication. For example, Aas [31] reported that patients with childhood traumatic schizophrenia have more severe clinical symptoms, and the improvement rate after treatment is reduced. Schneeberger's [32] research suggests that childhood Patients with traumatic schizophrenia need to use more types and larger doses of antipsychotic drugs and mood stabilizers in the treatment. In addition, childhood trauma is associated with impaired working memory, executive function, speech learning, and attention in people with schizophrenia, including those at very high risk of developing psychosis [33].

Therefore, in general, childhood trauma will increase the risk of schizophrenia and affect the clinical symptoms and prognosis of schizophrenia.

\section{SOCIOECONOMIC STATUS OF PARENTS}

Socioeconomic status (SES) refers to a measure of one's combined economic and social status and tends to be positively associated with better health. Income/social class, occupation, and education are the three most common factors needed to be considered [34]. Although it seems like socioeconomic status of parents has a little or indirect influence on mental disorders, poor socioeconomic status is alike to the chronic poisoning that unconsciously plays an adverse role in the development of schizophrenia. As for the economic situation of a family, the Family Stress Model (FSM) is one of the most persuasive models that describes a process by which the experience of severe economic pressure undermines parents' mental health and parenting and subsequent child adjustment [35]. Regarding the FSM, economic hardship will generate economic pressure on breadwinners in families and psychological distress will be subsequently disclosed to themselves. In the following stages, distress tends to ruin the interparental relationships and disrupt the parenting of children, which could lead to parents' affection, attention or care withdrawal from their children. At the end, the better the economic situation family has the more effective the parenting and the positive the children's mental health outcomes [36]. On the ground of Danish cohort study with more than 1 million participants, researchers proposed that lower parental income during childhood predicted a significantly greater risk for developing schizophrenia in adulthood and the more time that children live with poor family economic situations, the greater the risk [37].

Except for the analysis from the perspective of economics, the literacy or education background of parents can also be a factor in determining the level of income which will stratify people with higher SES from lower SES. Even though most SES data are consistent with little or no effect at birth, a gradual onset of subtle educational and occupational disabilities is associated with an eventual diagnosis of schizophrenia [38]. Mental health literacy (MHL) indicates the knowledge of how to prevent a mental disorder; recognition of disorders when developing; knowledge of effective self-help strategies for mild-to-moderate problems; and 
first aid skills to help others [39]. This capability is, indeed, the one that everyone needs, but higher MHL is even more supportive and powerful when there is a patient with chronic mental illness such as schizophrenia in the family. Increased sensitivity or awareness of the disorder is a psycho-educational component that not only allows the family to commit to recovery therapy but also alleviates caregiver's anxiety. For the sake of patients with schizophrenia, parents are able to facilitate understanding of the disorder and therefore, assist patients to facilitate behavior modification or even conduct some self-treatments within their abilities that will benefit their children with schizophrenia [40].

\section{CONCLUSION}

In this paper, we analyze the parental/family impacts on schizophrenia through five aspects: parents' socioeconomic status, genetic inheritance, personality traits, parenting style, and childhood trauma. Inherited genes and personality traits, as well as gene mutations, determine the feasibility of being diagnosed with schizophrenia. Family is where individual personality's formation and development take place. Meanwhile, being the most powerful indirect factor in developing schizophrenia or causing the ultimate confirmed diagnosis, socioeconomic status of parents (including income, education, etc.) is clearly related to the mental illness. The poorer the economic situation of family the greater risk of being diagnosed with schizophrenia or even exacerbating the original conditions. Similarly, parents with the higher level of education/mental health literacy can enhance their understanding of schizophrenia and therefore, give appropriate support to their children to achieve the management or prevention of schizophrenia. Family disharmony and inappropriate educational methods affect individual personality's formation, which leads to the occurrence of schizophrenia and related mental illnesses. Family factors of schizophrenia include parenting style and childhood trauma. Poor parenting styles may increase the likelihood of individuals suffering from schizophrenia. And childhood trauma will not only increase the incidence of having schizophrenia, but also its recovery. Environmental factors may actually be created under the drive of genes. The environment easily induces internal genes, leading to schizophrenia. In general, family is an important factor that needs to be considered in the cause analysis and intervention of schizophrenia.

Overall, all the factors that are relevant to parents can cumulatively and negatively affect schizophrenia like genes that parents carry, which could directly affect their offspring's morbidity rate. Nevertheless, patients with schizophrenia will be greatly recovered as well if their parents or other family members are well educated themselves with a deeper understanding of this mental disorder. This review benefits the public that they could procure general knowledge about parental influences in Schizophrenia. But, according to the preliminary findings related to parenting patterns, we have found that the relationship between paternal parenting and the advent of schizophrenia during childhood has not been well underlined and went unheeded. The association between fathers and the development of schizophrenia is unclear so far. At the meantime, we expect more research regarding patients' personality similarity, aside from the conclusions that were drew from TCI, could be conducted. In the future, we hope this paper can draw the attention of the research community to the paternal parenting's attribution to schizophrenia and leads to the creation of systematic supporting guideline for family members to assist patients as well as the increasing promotion of psycho-education about schizophrenia.

\section{REFERENCES}

[1] American Psychiatric Association. (2013). Schizophrenia spectrum and other psychotic disorders. In Diagnostic and statistical manual of mental disorders (5th ed.). https://doi.org/10.1176/appi.books.9780890425596 .dsm05

[2] Schizophrenia. (2019, October 4). World Health Organization.

https://www.who.int/news-room/fact-sheets/detail/ schizophrenia

[3] Insel, T. R. (2010). Rethinking schizophrenia. Nature, 468(7321), 187-193. https://doi.org/10.1038/nature09552

[4] Tsuang, M. (2000). Schizophrenia: genes and environment. Biological Psychiatry, 47(3), 210 220.

https://doi.org/10.1016/s0006-3223(99)00289-9

[5] Stilo, S. A., \& Murray, R. M. (2019). Non-Genetic Factors in Schizophrenia. Current Psychiatry Reports, 21(10), https://doi.org/10.1007/s11920-019-1091-3

[6] Costa E Silva JA, Steffen RE. Urban environment and psychiatric disorders: a review of the neuroscience and biology. Metabolism. 2019 Nov;100S:153940. doi: 10.1016/j.metabol.2019.07.004. PMID: 31610855.

[7] Davis, J., Eyre, H., Jacka, F. N., Dodd, S., Dean, O., McEwen, S., Debnath, M., McGrath, J., Maes, M., Amminger, P., McGorry, P. D., Pantelis, C., \& Berk, M. (2016). A review of vulnerability and risks for schizophrenia: Beyond the two hit hypothesis. Neuroscience \& Biobehavioral 
Reviews, 65, 185-194. https://doi.org/10.1016/ j.neubiorev.2016.03.017

[8] Andreasen NC, Olsen S. Negative v Positive Schizophrenia: Definition and Validation. Arch Gen Psychiatry. 1982;39(7):789-794. doi:10.1001/archpsyc.1982.04290070025006

[9] Robert W. Buchanan, Persistent Negative Symptoms in Schizophrenia: An Overview, Schizophrenia Bulletin, Volume 33, Issue 4, July 2007, Pages 1013-1022, https://doi.org/10.1093/schbul/sbl057

[10] Targum SD, Keefe RS. Cognition and schizophrenia: is there a role for cognitive assessments in diagnosis and treatment? Psychiatry (Edgmont). 2008 Dec;5(12):55-9. PMID: 19724777; PMCID: PMC2729617.

[11] Francisco J. Vaz, M.D., Ph.D., Augustín Béjar, M.D., Ph.D., Mariano Casado, M.D., Ph.D., Insight, Psychopathology, and Interpersonal Relationships in Schizophrenia, Schizophrenia Bulletin, Volume 28, Issue 2, 2002, Pages 311317 ,

https://doi.org/10.1093/oxfordjournals.schbul.a006 940

[12] Wayne S. Fenton, M.D., Evolving Perspectives on Individual Psychotherapy for Schizophrenia, Schizophrenia Bulletin, Volume 26, Issue 1, 2000, Pages 47-72, https://doi.org/10.1093/ oxfordjournals.schbul.a033445

[13] Bustillo, J. R., Lauriello, J., Horan, W. P., \& Keith, S. J. (2001). The Psychosocial Treatment of Schizophrenia: An Update. American Journal of Psychiatry, 158(2), $163-175$. https://doi.org/10.1176/appi.ajp.158.2.163

[14] Robert S. Kern, Shirley M. Glynn, William P. Horan, Stephen R. Marder, Psychosocial Treatments to Promote Functional Recovery in Schizophrenia, Schizophrenia Bulletin, Volume 35, Issue 2, March 2009, Pages 347-361, https://doi.org/10.1093/schbul/sbn177

[15] McFarlane, W. R. (2016). Family Interventions for Schizophrenia and the Psychoses: A Review. Family Process, 55(3), 460-482. https://doi.org/10.1111/famp.12235

[16] Sullivan PF, Kendler KS, Neale MC. Schizophrenia as a complex trait: evidence from a meta-analysis of twin studies. Arch Gen Psychiatry. 2003;60:1187.

[17] THE GENETIC THEORY OF SCHIZOPHRENIA I American Journal of Psychiatry. (2020). American Journal of Psychiatry. https://ajp.psychiatryonline.org/doi/abs/10.1176/ajp .103.3.309

[18] Stefansson H, Sigurdsson E, Steinthorsdottir V, Bjornsdottir S, Sigmundsson $\mathrm{T}$, Ghosh $\mathrm{S}$ et al. Neuregulin 1 and susceptibility to schizophrenia. Am J Hum Genet 2002; 71: 877-892.

[19] Munafò, M. R., Thiselton, D. L., Clark, T. G., \& Flint, J. (2006). Association of the NRG1 gene and schizophrenia: a meta-analysis. Molecular Psychiatry, 11(6), 539-546. https://doi.org/10.1038/sj.mp.4001817

[20] Farrell, M., Werge, T., Sklar, P. et al. Evaluating historical candidate genes for schizophrenia. Mol Psychiatry 20, 555-562 (2015). https://doi.org/10.1038/mp.2015.16

[21] Increase in harm avoidance by genetic loading of schizophrenia. (2012). Comprehensive Psychiatry, 53(4), 372-378. https://doi.org/10.1016/j.comppsych.2011.05.004

[22] Thimm, J. C. . (2010). Mediation of early maladaptive schemas between perceptions of parental rearing style and personality disorder symptoms. J Behav Ther Exp Psychiatry, 41(1), 52-59.

[23] Zhang, Z. , Wu, D. , Zhang, Z. , \& Hospital, S. . (2014). Porental rearing style of patient with mental disorders. China Journal of Health Psychology.

[24] Zhang, X. , Liu, L. , Lai, H. , Zheng, T. , Psychiatry, D. O. , \& Hospital, W. C. , et al. (2017). The relationship of self-efficacy and coping styles with parenting styles in patients with schizophrenia. West China Medical Journal.

[25] Otani, K. , Suzuki, A. , Oshino, S. , Ishii, G. , \& Matsumoto, Y. . (2009). Effects of the "affectionless control" parenting style on personality traits in healthy subjects. Psychiatry Research, 165(1-2), 181-186.

[26] Baker, C. N. , \& Hoerger, M. . (2012). Parental child-rearing strategies influence self-regulation, socio-emotional adjustment, and psychopathology in early adulthood: evidence from a retrospective cohort study. Pers Individ Dif, 52(7), 800-805.

[27] Hui, G. C. , Huang, Y. , Liu, Z. , \& Liu, B. . (2011). Associations linking parenting styles and offspring personality disorder are moderated by parental personality disorder, evidence from china. Psychiatry Res, 189(1), 105-109.

[28] Filippo, V. , Feikje, S. , Marjan, D. , Ritsaert, L. , Tineke, L. , \& Wolfgang, V. , et al. (2012). 
Childhood adversities increase the risk of psychosis: a meta-analysis of patient-control, prospective- and cross-sectional cohort studies. Schizophrenia Bulletin(4), 661-671.

[29] Xie, P. , Wu, K. , Zheng, Y., Guo, Y. , Yang, Y. , \& He, J., et al. (2017). Prevalence of childhood trauma and correlations between childhood trauma, suicidal ideation, and social support in patients with depression, bipolar disorder, and schizophrenia in southern china. J Affect Disord, 41-48.

[30] Bruni, A. , Carbone, E. A., Pugliese, V., Aloi, M. , G Calabrò, \& Cerminara, G. , et al. (2018). Childhood adversities are different in schizophrenic spectrum disorders, bipolar disorder and major depressive disorder. Bmc Psychiatry, 18(1).

[31] Aas, M. , Andreassen, O. A. , Aminoff, S. R. , Frden, A. , Romm, K. L. , \& Nesvg, R., et al. (2016). A history of childhood trauma is associated with slower improvement rates: findings from a one-year follow-up study of patients with a first-episode psychosis. BMC Psychiatry, 16(1), 126.

[32] Schneeberger, A. R. , Muenzenmaier, K. , Castille, D. , Battaglia, J. , \& Link, B. . (2014). Use of psychotropic medication groups in people with severe mental illness and stressful childhood experiences. Journal of Trauma \& Dissociation, 15(4), 494-511.

[33] Popovic, D., Schmitt, A., Kaurani, L., Senner, F., Papiol, S., Malchow, B., Fischer, A., Schulze, T. G., Koutsouleris, N., \& Falkai, P. (2019). Childhood Trauma in Schizophrenia: Current Findings and Research Perspectives. Frontiers in neuroscience, 13, 274.

[34] Baker, E. H. (2014). Socioeconomic Status, Definition. The Wiley Blackwell Encyclopedia of Health, Illness, Behavior, and Society, 2210-2214. https://doi.org/10.1002/9781118410868.wbehibs39 5

[35] Scaramella, L. V., Sohr-Preston, S. L., Callahan, K. L., \& Mirabile, S. P. (2008). A Test of the Family Stress Model on Toddler-Aged Children's Adjustment Among Hurricane Katrina Impacted and Nonimpacted Low-Income Families. Journal of Clinical Child \& Adolescent Psychology, 37(3), 530-541. https://doi.org/10.1080/15374410802148202

[36] Masarik, A. S., \& Conger, R. D. (2017). Stress and child development: a review of the Family Stress
Model. Current Opinion in Psychology, 13, 85-90. https://doi.org/10.1016/j.copsyc.2016.05.008

[37] Hakulinen C, Webb RT, Pedersen CB, Agerbo E, Mok PLH. Association Between Parental Income During Childhood and Risk of Schizophrenia Later in Life. JAMA Psychiatry. 2020;77(1):17-24. doi:10.1001/jamapsychiatry.2019.2299

[38] Carles Muntaner, William W. Eaton, Richard Miech, Patricia O’Campo, Socioeconomic Position and Major Mental Disorders, Epidemiologic Reviews, Volume 26, Issue 1, July 2004, Pages 53-62, https://doi.org/10.1093/epirev/mxh001

[39] Kutcher, S., Wei, Y., \& Coniglio, C. (2016). Mental Health Literacy: Past, Present, and Future. The Canadian Journal of Psychiatry, 61(3), 154

158. https://doi.org/10.1177/0706743715616609

[40] Merinder, L., Viuff, A \& Langesen, H. (1999). Patient and relative education in community psychiatry: a randomized controlled trial regarding its effectiveness. Social Psychiatry and Psychiatric Epidemiology, 34, 287-294. 